\title{
$\mathbf{X}$. \\ Kleinere Mittheilungen.
}

1.

\section{Ein Beitrag zur Diagnose der Ohrenkrankheiten.}

\author{
Von Dr. Z. Oppenheimer, a. o. Professor in Heidelberg.
}

Bei der Untersuchung zweier Schwerhörigen fiel es mir auf, dass sie gewisse Worte leichter verstanden als andere. Eine aufmerhsame Beobaclitung führte mich darauf, dass alle Worte', welche die Vokale a und o enthielten, verhältnissmässig leicht verstanden, dass hingegen $e$ braum und $i$ gar nicht wahrgenommen wurde. Die bierzu angestellten Versuche wurden selbstverständlich so gemacht, dass die Kranken meinen Mund nicht sehen konnten und der Ton in allen Worten, welche mit einander verglichen werden sollten, soviel als möglich gleich stark gesprochen wurde. Leicht wurden so verstanden die Worte: acht mal acht, gar nicht die Worte "vier und vierzig“, „ich versichre Sie". Forderte man die Kranken auf, das zu wiederholen, was sie verstanden hatten, so geschah dies häufig vollkommen richtig, wenn der Sinn des Sátzes ein einfacher war. Nabm ich aber einen lângeren Satz mit Ausdrücken, welche den Kranken fremd waren, z. B. einen lateinischen Krankheitsnamen, so wiederholten sie nur die Silben, in welchen a und o als Vokale vorkamen, die anderen nicbt.

Was nun die weitere Beschaffenheit des Gehörs dieser Kranken betrifft, so fand sich bei der ersten, Fräulein W., 45 Jahre alt, eine Schwerhörigkeit seit 20 Jahren. Der äussere Gehörgang zeigte keine Anomalie, das Trommelfell war matt, gräulich. Die Tuba war durchgängig und ein Luftstrom ging mit etwas Rasseln, aber leicht und frei in die Paukenhöhle. Die Schleimhaut des Pharyox ist geröthet und mit erweiterten Venen durchzogen; auch zeigt sich daselbst eine reichliche Schleimsecretion. Die Kranke hatte ein lebhaftes Colorit des Gesichts, Iitt an Wallungen zum Kopf und klagte über häufige leichte Schwindelanfälle. Die Sprache verstand sie, wie oben angegeben, aber nur bei sehr lautem Sprechen. Die Uhr wurde kanm auf $\frac{1}{4}-\frac{1}{2}$ Zoll Entfernung vom rechten Ohre gehört, auf dem linken nar, wenn man sie hart an das Ohr andrỉckte.

Die zweite, Fräulein L., 16 Jahre alt, zeigte weder am Trommelfell nocb an der Tuba eine Veränderung. Die Ubr wurde in $5-6$ Zoll Entfernung vom Obre gehört. Hingegen war das Verständniss der Sprache schwieriger als bei der ersten. Man musste schon ziemlich schreien, und die Kranke musste den Mund genan sehen, um eine Unterhaltung führen zu können. Dadurch erhielt diese Schwer- 


\section{1}

hörige jenen ängstlich lauernden Gesichtsausdruck, den wir bäufig bei Scbwerhörigen sehen.

Bei beiden Kranlien stelite ich anfänglich die Diagnose auf Katarrh des mittleren 0hres and behandelte sie demgemäss. Obgleich nun die Behandlung bei Beiden den Erfolg hatte, dass sie besser zu hören glaubten, dass die Ulr in grösserer Entfernung vom Ohre als früher wabrgenommen wurde, so überzeugte ich mich doch nach einigen Wochen, dass der Erfolg nur ein scheinbarer war, indem das Verständaiss der Sprache gar nicht wesentlich gebessert wurde. Bei der zweiten Patientin war mir dies um so auffallender, weil ich im Mittelohre nur einen unbedeutenden Katarrh annehmen konnte und mit der Besserung des Katarrhs die Wahrnehmung des Tiktaks der Uhr erleichtert warde. Bei der ersten Kranken, wo gar kein Zweifel an dem ausgedehnten Katarrh bestehen konnte, war sicherlich ebenfalls eine Besserung desselben eingetreten, aber die Sprache blieb unverstanden, wie oben angegeben wurde, wenn man auch vielleicht weniger laut zu sprechen genōthigt war. Allein auf letateren Umstand kann man hein Gewicht legen, da es wohl unmöglich ist, in längeren $Z$ wischenräumen die Stärke der Stimme zu vergleichen.

Die Kranken verliessen die ärztliche Behandlung und ich Labe seitdem keine Nachrichten über ihr Gehörvermögen erhalten können. Um so mehr war ich aber bestrebt, eine Erklärung für diese räthselhafte Erscheinung zu ernitteln. Bei dem Studium des neuen Werkes von Helmholtz ,die Lehre von den Tonempfindungen" fand ich endlich, wie mir scheint, den gewünsclrten Aufschluss. Der geniale Physiologe Kommt daselbst zu dem Schlusse, dass im Gehörorgane zwei verschiedene Theile zur Empfindung der Luftbewegung vorhanden sind. Die nervösen Endorgane des Vorhofs und der Bogengänge empfinden die nicht periodischen Bewegungen der Luft, die Geräusche, während die Endorgane in der Schneche, die Corti'schen Fasern die Empfindung der periodischen Bewegungen der Luft, die musikalischen Töne aufnehmen. Helmholtz schliesst dann weiter, dass dic einzelnen elastischen Fasern in der Corti'schen Membran die Empfindung bestimmter Töne möglich machen und dass verschiedene Töne verschiedene Nervenfasern erregen werden: „Die Empfindung der Klangfarbe würde darauf beruben, dass ein Klang ausser den seinem Grundton entsprechenden Corti'schen Fasern noch cine Anzahl anderer in Bewegung setzte, also in mebreren verschiedenen Gruppen von Nervenfasern Empfindungen erregte". Die letzte Ansicht findet hauptsüchlich darin ibre Stütze, dass Helmboltz das Gesetz aufgestellt und bewiesen hat, dass die klangfarbe des musikalischen Theils eines Klanges nur abhängt von der Zahl und Stärke der Theiltöne und nicht von ihren Phasenunterschieden. Es gilt dieses Gesetz nicht nur bei den musikalischen Instrumenten, sondern ganz bestimmt auch von den Vokalen der menschlichen Stimme, in welchen mit voller Evidenz die Verstärkung einzelner Obertöne durch die Resonanz der Mundböhle nachgewiesen wurde.

Diese glänzenden Untersuchungen auf dem Gebiete der Physiologie sind sogleich fruchthringend, wie man sie auf die Pathologie überträgt. Sie bringen Licht in das dunkle Gebiet der sogenannten nervösen Schwerhörigkeit, in jene Erlirankungen des Labyrinths, welche man beim Lebenden wohl vermuthen, aber nicht Archiv f. pathol. Anat, Bd, XXX. Uft, 1 u. 2. 
diagnosticiren kann, welche v. Tröltsch als die Krankheiten definirt, bei denen die Kranken Nichts hören und die Aerzte Nichts sehen.

Zuerst lässt sich erwarten, dass bei sonst gesundem Zuleiten der Apparat des Gebörorgans eine Veränderung in dem bäutigen Theile des Vorbofes und den halbzirkelförmigen Kanälen eine Beeinträchtigung oder Vernichtung des Vermögens Geräusche zu empfinden, nach sich zieben muss.

Zweitens wird jede Läsion in dem häutigen Theile der Scbnecke die Empfindung der musikalischen Töne beeinträchtigen oder vernichten.

Die Richtigkeit dieser Hypothese würde sich nur dadurch heweisen lassen, dass man in gut beobacbteten Fällen eine pathologische Veränderung eines einzigen Organs nachweisen könnte. Leider kann bis heute die pathologische Anatomie des Gehörorgans noch nicht den gewünschten Aufschluss geben. Denn einmal sind die Yeränderungen eines beschränkten Theils im Innern des Ohres selten. Es ist begreiflich, dass bei der Nähe der einzelnen Theile des Gehörs die Krankheiten sich leicht ausbreiten und in benachbarten Theilen störungen verursachen. Dann ist die Untersuchung der einzelnen Weichtheile äusserst schwierig, und wenn auch eine sehr geschickte Hand die Knochen entfernt und die Weichtheile in ihrer ganzen Integrität zur Untersuchung blossgelegt hat, so ist die milsroskopische Untersuchung bei dem complicirten Bau der betreffenden Gewehe noch so beträchtich erschwert, dass kaum ein in allen Beziehungen richtiges Resultat erwartet werden kann. Endlich muss hier ein Vorwurf wiederholt werden, den Voltolini mit vielem Rechte betont, dass nämlich in den meisten uns mitgetheilten Sectionsberichten über erkrankte Gehörorgane die Erscheinungen während des Lebens nicht gekannt oder nicht mitgetheilt wurden. Aus diesen lässt sich daher kein Schluss auf die Function der einzelnen Theile des Gehörs ziehen. Voltolini hat daher vollkommen Recht, dass er solche Früehte zwar fïr werthvoll in Bezug auf pathologische Adatomic, aber für nutzlos in Bezug auf klinische Auffassung erklärt.

Unter diesen Umständen ist es begreiflicherweise äusserst misslich, die oben aufgestellte Hypothese zu beweisen. Es ist mir nur gelungen, zwei Fälle in der Literatur aufzufinden, welche hier erwähnt zu werden verdienen.

Voltolini berichtet (Sectionen an Schwerhörigen. Virchow's Archiv Bd.XXII. S. 110. Fall 1) von einer 68jährigen Frau, dass sie auf dem rechten Ohre ganz taub, auf dem linken die Uhr auf $\frac{1}{2}$ Zoll Entfernung hörte. Bei der Section fand sich auf dem rechten Ohre vollständige Verknöcherung der Fenestra ovalis und Verwachsung der Fenestra rotunda. Die Säckchen des Vorhofs sind zum Theil mit 0 tolithen ganz vollgestopft, zum Theil in eine gelbe Masse (verdicktes Bindegewebe) verwandelt. Der Anfang der Lamina spiralis zeigt ein milchweisses Aussehen. Die Basis stapedis prominirt stark in das Vestibulum und ist vollständig fest mit der Wand des Vorhofs verwachsen. In der Schnecke sonst keine "wesentliche“ Veränderung. Der Gehörnerv zeigt amyloide Degeneration.

In dem linken Obre (Hörweite $\frac{1}{2}$ Zoll) zeigte sich Fenestra rotunda, die Bogengänge und der Hörner wie auf dem rechten ohre verändert, hingegen im Vestibulum sind die Säckchen verdickt, gallertartig, mit zabllosen 0 to- 
lithen. Die Fenestra ovalis mit der Basis stapedis bietet ausser einer Schwerbeweglichkeit des Steigbügels nichts Abnormes. Die Schnecke frei.

Voltolini erklärt nun die absolute Taubheit auf dem rechten Ohre für eino Folge der vollständigen knöchernen Verwachsung der Fenestra ovalis. Meiner Meinung nach erklärt dieser Befund die absolute Taubleit nicht. Man mus wohl eine Schwerhörigkeit als Folge der Eïwachsung der Basis stapedis in die Fenestra ovalis annebmen, weil die Sclsallleitung erschwert ist. Aber aufgeboben kann sie dadurch nicht vollständig werden, weil auch Knochen den Schall leiten, und die Schallwellen sich dann auf die Weichtheile übertragen. Wären nun die Weichtheile in diesem Falle intact gewesen, so bätte noch ein Ton oder Gerüuschempinndung eintreten müssen. Ja aber die Weichtheile in Vorhof wesentlich verändert waren, so scheint es wohl walirscheinlicher, dass diese Verónderungen die Ursache der Taubbeit waren. Voltolini spricbt dies selbst an einer anderen Stelle mit den Worten ans „Soviel steht fest, wo wir absolute Taublheit finden, muss das Labyrinth und der Gehörnerv der Sitz des Leidens sein, da weder eine Af́ection des äusseren Gehörganges noch der Tuba noch der Paukenhöhle absolute Taubheic bedingt. ". (Abhandlungen der schles. Gesellsch. für vaterl. Cultur. Abth. für Naturwissensch. und Med. 1862. Heft 1.)

Wir erklären hiernach diesen Fall in der Art, dass auf dem rechten Ohre durch die vollständige Umänderung der Säckchen des Vorhofs das Vermögen Gcrăusche wahrzunebmen aufgehoben war. Auf dem linken $0 \mathrm{hr}$ war diese Läsion noch nicht so weit gediehen, und daher bestand hier noch das Vermögen, dio Uhr auf $\frac{1}{2}$ Zoll Entfernung za hüren - eine Entfernung, welche bei der Bewcglichkeit der Gehörknöchelchen doch verhältnissmässig sehr unbedeutend ist.

Wie erklärt sich aber das mangelnde Verstündniss der Sprache, die Taubhcit für die Sprache auf beiden Ohren, da die Schnecke lieine wesentliche Veränderung gezeigt hat? Hier kommt die Bedeutung der Fenestra rotunda wesentlich in $\mathrm{B}$ tracht. Die durch den Schall erzeugte Erschitterung des Labyrinthwassers rerursacht entweder ein Ueberfliessen durch das Helicotrema in die Paukentreppe der Scbnecke, oder die membranöse Scheidewand wird gegen die Paukentreppe hin verdrängt. In beiden Fällen wird das runde Fenster sich gegen die Paukenböhle zu hervordrängen müssen. Wenn nun, wie in dem angeführten Falle, das runde Fenster verwachsen ist - nach Voltolini's Besclireibung "war die Oeffnung des Kanals, der zum Foramen fübrt, vollständig durch eine falsche Membran verdeclst, d. b. überspannt; diese jst dick concav; zieht man sie ab, so muss man sich durch Bindegewebsfasern und Bänder bis zum Foramen rotundum durcharbeiten" so ist eine Bewegung des Schneckenwassers und damit eine Erregung der Cortíschen Fasern unmöglich. Wegen der angeführten Bedeutung des runden Fensters dürfte es wohl zweckmässig seiu, dasselbe als einen Theil der Sclunecke und nicht als zur Paukenhölle gehörig zu betrachten.

Ein zweiter bieher bezüglicher Fall betraf einen Musikus, welcher einen flbromuskulären Tumor in der Cupula der Schnecke hatte, welcher diese ganz ausfüllte (s. Voltolini a. a. 0. S. 24). Das ganze übrige Ohr des Kranlien war gesund. „Die Frau des Kranken gab an, dass er schwerlı̈̈rig war und besonders, 
wenn ihm kalt wurde, sofort über Sausen und Schwerhörigkeit klagte und erwähnt wiederbolt des merkwürdigen Umstandes, dass jedesmal, wenn er üher Schwerhörigkeit liagte, auch seine Sprache schlechter und unverständlicher wurde." Leider gibt Voltolini nicht an, wie sich das scheinhar gesunde Ohr verhalten hat. Es lässt sich wohl vermuthẹn, dass es nicht ganz gesund war, da im anderen Falle die Schwerhörigkeit sich schwer erklären liesse. In bohem Grade der Reachtung werth ist aber, dass die Sprache zuweilen unverständlich wurde, weil dies beweist, dass dem Kranken dann die Fähigkeit abging, seine Mundhöhle so abzustimmen, dass die Obertöne die richtige Verstärkung bei der Bildung der Vokale erfahren konnten. Dieser Mangel konnte nur dadurch entstehen, dass er selbst die Vokale nicht hörte, weil seine Schnecke krankhaft entartet war.

So gering auch die Zabl der hiehergehörigen Fälle ist und so schwach auch ibre Beweiskraft ist, so habe ich dieselben doch nicht unerwähnt lassen wollen und will nun noch eine Beobachtung mittheilen, die schon während des Lebens fïr das Vorhandensein zweier verschiedener Empfindungsorgane im Ohre spricht. Man hat nämlich häufig beobachtet, dass Schwerhörige die Uhr noch ziemlich gut hörten und die Sprache nicht verstanden oder auch ungekehrt. Besonders auffallend ist es, dass im Laufe der Bebandlung von Obrenkrankheiten die Wabmehmung der Geräusche sich häufig bessert und die Sprache wie früher undeutlich bleibt. Bis jetzt hat man diese scheinbar paradoxe Erscheinung nicht zu erklären verstanden. Sobald man aber zwei verschiedene Empfindungsorgane nach Hel mholtz annimmt, so wird der Sinn der Erscheinung klar, und sie selbst ein werthvolles diagnostisches Zeichen, insofern man dadurch im. Stande ist, bald auf ein Leiden des Vorhofs und der Fenestra ovalis, bald auf ein Leiden der Schnecke und der Fenestra rotunda za schliessen. $O b$ in dem ersten Falle der Vorhof oder die Fenestra ovalis mit dem Steigbügel, oder in dem anderen Falle die Schnecke oder die Fenestra rotunda die ursprünglich erkrankten Theile sind, wird sich aus der Anamnese und hauptsächlich aus dem Vorhandensein eines chronischen Katarrhs des Mittelobres ergeben.

Wenn nun die aufgestellte Hypothese durch die mitgetheilten Beobachtungen eine grössere Wabrscheinlichkeit gewonnen hat, so wird man auch weiter schliessen können, dass Veränderungen in der Schnecke selbst die Wahrnehmung von Luftschwingungen in verschiedener Weise modificiren können. Eine vollständige Zerstörung der Corti'schen Membran wird absolute Taubheit für Töne erzeugen. Aber eine nur theilweise die Corti'sche Membran treffende pathologische Veränderung wird auch nur theilweise die Gehörsempfindung aufheben. Jedem Arzte ist es bekannt, dass manche Individuen nur für bobe Töne taub sind, wälırend die Empfindung tiefer Töne entweder normal oder nur geschwächt ist. Es gehört ferner hierher die äusserst merkwürdige Beobachtung v. Wittich's (Königsberger med. Jahrbücher 1861. Bd.3. S. 40-45). v. Wittich hörte, nachdem er \& Wochen früher von einer beftigen Entzündung des mittleren und später auch des äusseren Ohres befallen worden, alle Töne der eingestrichenen Octave verschieden und zwar auf dem kranken ohre einen halben Ton höher als auf dem gesunden. Dabei war die Wahrnehmung der Geräusche wohl etwas geschwächt, aber nicht aufge- 
hoben. Er hörte das Picken der Taschenuhr mit dem kranken Obre, wenn er das Zifferblatt dicht an die Ohrmuschel anlegte. Das Anschlagen des Klöpfels einer schwach tönenden Tischglocke hörte er in einer Entfernung von $1 \frac{1}{2}$ Fuss, während er das Klingen erst wenige Zoll vom Ohre wabrnahm. Die Erklärung dieser Erscheinung des Doppelthörens kann nur dadurch gegeben werden, dass man eine circumscripte Schwellung in der Corti'schen Membran annimmt. Da die elastíschen Fasern in der Corti'schen Membran nur gemäss ihrer Abstimmung durch einen Ton in Schwingung versetzt werden; so müssen bei einer durch Schwellung der Fasern bedingten Verstimmung auch andere Fasern durch einen Ton schwingen als unter normalen Verhältnissen. Nimmt man z. B. an, die Fasern, welche dem Tone B entsprechen, seien so verstimmt, dass sie nur durch den Ton. $A$ in Mitschwingung versetzt werden können, also um einen balben Ton tiefer gestimmt sind alș gewöhnlich, so wird ein Ton B diese Fasern nicht mehr erregen, sondern nur noch der Ton A. Unsere Nerven empfinden aber diese durch den Ton A erregten Schwingungen nicht als A, sondern ibrer Gewohnheit gemäss als B und so erklärt es sich, dass das kranke 0 hr die Töne in den oben angegebenen Grezzen einen balben Ton höher hörte, als das gesunde.

Endlich ist der in den oben angefülirten Fällen vorhandene Mangel, die Vokale zo verstehen, nur dadurch zu erklären, dass in der Corti'schen Membran ein Theil der Fasern zu Grunde gegangen ist und zwar hauptsächlich diejenigen, welche zur Empfindung der hohen verstärkten Obertöne dienen. Will man andere Momente zur Erklärung aufsuchen, so würden diese wohl die Schwerbörigkeit im Algemeinen erklären, aber nicht den Mangel der Perception èinzelner Vokale. Die Kiranken litten an Katarrh des Mittelohres, sie hörten anfänglich die Uhr schlecht. Diese Zustände aber besserten sich; die Beweglichkeil der Gelörknöchelchen, das Verhaiten des Vorhofs muss daher ziemlich normal gewesen sein, und es bleibt nur übrig eine Anomalie in der Schnecke aufzusuchen, welche sich aus den physiologischen Beobachtungen als wahrscheinlich voraussetzen lässt. Es liegt nahe, diesen Zustand des Mangels der Perception der Vokale mit der Farbenhlindheit des Auges in Analogie zu bringen. Man müsste eine Klangfarbentaubheit anfstellen. Die Analogie ist aber deshalb unrichtig, weil bei der Farbenblindheit der Hypothese nach ein Theil der Nerven fehlt, während hier ein Theil des schalleitenden Apparates zu Grunde gegangen ist.

Zuletzt bleibt mir noch übrig, die aufgestellte Hypothese, welche wegen ihrer Uebereinstimmung mit den physiologischen Thatsachen einen hohen Grad von Wahrscheinlichkeit besitzt, der Prüfung durch die Ohrenärzte zu empfehlen. Hierzụ wïrde die Anwendung von solchen Instrumenten geeignet sein, welche einfache Töne in grosser Anzahl hervorbringen lassen, wie die. Prüfung des Gehörs mittelst einer grossen Zahl von Stimmgabeln oder mittelst einer vervollkommeten Glasharmonika, die man mit einer grösseren Zabl gut gestimmter Gläser versehen müsste. Die in dieser Weise angestellten Beobachtungen in Verbindung mit einer genauen pathologisch-anatomischen Untersuchung könnten das dunkle Gebiet der Labyrintherkrankungen aufklären. 